Ann. Zootech., 1984, 33 (2), 255-262

\title{
Note
}

\section{Influence de la modification de la fréquence de distribution des repas sur la digestibilité du régime alimentaire chez la truite arc-en-ciel}

\author{
G. CHOUBERT, J.M. BLANC * et P. LUQUET \\ I.N.R.A., Laboratoire de Nutrition et d'Elevage des Poissons \\ * Laboratoire d'Ecologie des Poissons et d'Aménagement des Pêches \\ Centre de Recherches hydrobiologiques, \\ Saint-Pée-sur-Nivelle, F 64310 Ascain
}

\begin{abstract}
Résumé
Les valeurs des CUD de la matière sèche et de l'azote obtenues à partir de truites recevant un et deux repas par jour (pour un même taux d'ingestion, 1 p. 100 poids vif/jour) ne diffèrent pas significativement.

Le changement de fréquence de distribution de l'aliment n'entraîne aucune évolution systématique des CUD dans les jours qui suivent.

Le choix de l'une ou l'autre de ces fréquences de distribution de l'aliment n'est donc qu'une question d'opportunité. Il convient toutefois de noter que les variations d'erreur (bac, jour), plus faibles dans le cas d'une fréquence de distribution d'un repas par jour, pourraient même la rendre préférable.
\end{abstract}

Mots clés : nutrition, fréquence, repas, digestibilité, truite.

\section{Introduction}

La normalisation de la fréquence de distribution des repas à retenir pour les études de digestibilité chez le poisson n'a fait, à l'heure actuelle, l'objet d'aucun travail expérimental. Selon les expérimentateurs, le nombre utilisé varie de un (Smith, 1971 ; Windell, Foltz \& Sarokon, 1978 ; Smith, Peterson \& Allred, 1980) à quatre repas (KAUSHIK \& LUQUET, 1976) pour la truite arc-en-ciel et de un (Pappas, Tiemeier \& Deyoe, 1973) à deux repas (Andrews, Murray \& Davis, 1978) pour le poisson chat. Certains auteurs utilisent même une distribution alimentaire en continu (Austreng, 1978).

Cet aspect méthodologique mérite cependant une attention particulière car il est connu que le nombre des repas conditionne la quantité d'aliment consommée (Possompes, 1973; Luouet, Renou \& KaushiK, 1981) et que celle-ci affecte l'effi- 
cacité de la digestion (Elliott, 1976 ; Windell, Foltz \& Sarokon, 1978 ; Bergot \& Breque, 1983). Une augmentation de la quantité ingérée, provoquée soit par une augmentation du nombre de repas soit par rationnement, à nombre de repas identique, entraîne une diminution de la valeur des coefficients d'utilisation digestive. Une explication peut être donnée par la diminution du temps de contact enzyme-substrat, car il a été montré que l'augmentation de la quantité d'aliment ingéré affecte la vitesse de transit du bol alimentaire, en diminuant le temps de rétention (CASTLE, 1956) ou le temps de transit moyen (ZiERLER, 1958 ; Possompes, BERgot \& Luquet, 1975). Ces derniers auteurs ont par ailleurs montré qu'après un changement du rythme alimentaire, dix jours sont nécessaires pour observer les courbes d'excrétion caractéristiques du nouveau rythme des repas. On ignore si au cours de cette période de transition l'efficacité de la digestion est affectée, comme cela est observé après un changement de température (CHOUberT, FAuconneAu \& LuQueT, 1982).

Le présent travail a donc pour objet d'étudier l'influence d'un changement de la fréquence de distribution des repas (un ou deux repas par jour) sur la digestibilité d'une même quantité d'aliment ingérée par la truite arc-en-ciel.

\section{Matériel et méthodes}

L'étude de l'utilisation digestive a été effectuée selon la méthode indirecte en incorporant de l'oxyde de chrome à l'aliment expérimental (EDIN, 1918) et en utilisant le dispositif automatique de récolte de fèces décrit par Choubert, De la Noue \& LuOUET (1982, 1983).

Les truites d'un poids moyen de $160 \mathrm{~g}$ sont réparties au hasard en douze lots de 15 poissons placés dans des bacs cylindroconiques de $40 \mathrm{~cm}$ de diamètre et d'une capacité utile de 50 litres. Chaque bac est alimenté en eau selon un débit de 4 litres/minute à une température maintenue à $13,5^{\circ} \pm 0,5^{\circ} \mathrm{C}$. L'aliment est distribué sous forme de granulé sec de $3,2 \mathrm{~mm}$ de diamètre à raison de $1 \mathrm{p} .100$ de leur poids vif par jour et selon le cas en 1 ou 2 repas (rythmes classiquement utilisés pour des truites d'une telle taille) aux heures suivantes : 1 repas/jour $=8 \mathrm{~h} 30$, 2 repas/jour $=8 \mathrm{~h} 30$ et $16 \mathrm{~h} 30$. Le plan expérimental est exposé dans le tableau 1. Après une période d'adaptation à un rythme d'alimentation durant 25 jours, une inversion de cette fréquence est effectuée; la partie expérimentale proprement dite correspond à la période de temps faisant suite au changement de fréquence de distribution de l'aliment. Elle s'étale sur une période de 10 jours, cette durée devant s'avérer suffisante pour mettre en évidence les éventuelles perturbations que ce changement pourrait entraîner (Possompes, Bergot \& Luouet, 1975). Chaque jour, les plateaux contenant les fèces sont changés et leur contenu lyophilisé, broyé et conservé au froid $\left(-18^{\circ} \mathrm{C}\right)$ en piluliers hermétiques jusqu'aux analyses.

Le dosage de l'azote total est effectué selon la méthode de Kjeldahl sur des prises d'essai de $400 \mathrm{mg}$ pour l'aliment et $50 \mathrm{mg}$ pour les fèces; la matière sèche des aliments est obtenue par chauffage à $95^{\circ} \mathrm{C}$ pendant $24 \mathrm{~h}$ sur une prise d'essai de $2 \mathrm{~g}$; l'oxyde de chrome est directement dosé sur des prises d'essai de $100 \mathrm{mg}$ par la méthode de Bolin, King \& Klosterman (1952). Chaque dosage est effectué en double. 


\section{Tableau 1}

Plan expérimental.

Experimental schedule.

\begin{tabular}{|c|c|c|c|c|c|}
\hline $\begin{array}{l}\text { Période } \\
\text { Period }\end{array}$ & $\begin{array}{c}\text { Durée (jours) } \\
\text { Duration } \\
\text { (days) }\end{array}$ & $\begin{array}{c}\text { Nature } \\
\text { de l'aliment } \\
\text { Type of feed }\end{array}$ & $\begin{array}{c}\text { Fréq } \\
\text { jourr } \\
\text { des } \\
\text { Daily } \\
\text { freqt }\end{array}$ & $\begin{array}{l}\text { lence } \\
\text { alière } \\
\text { epas } \\
\text { meal } \\
\text { ency }\end{array}$ & $\begin{array}{c}\text { Mesure de la } \\
\text { digestibilité } \\
\text { Digestibility } \\
\text { measurement }\end{array}$ \\
\hline $\begin{array}{l}\text { 1. Adaptation aux bacs } \\
\text { Adaptation to tanki }\end{array}$ & 20 & Commercial & $\begin{array}{c}\mathrm{A} \\
6 \mathrm{bacs} \\
1 \mathrm{R} / \mathrm{J}\end{array}$ & $\begin{array}{l}\mathrm{B} \\
6 \mathrm{bacs} \\
2 \mathrm{R} / \mathrm{J}\end{array}$ & \\
\hline $\begin{array}{l}\text { 2. Adaptation à l'ali- } \\
\text { ment expérimental } \\
\text { Adaptation to expe- } \\
\text { rimental feeds }\end{array}$ & 5 & $\begin{array}{l}\text { Expérimental } \\
\text { (93,5\% farine } \\
\text { de poisson) } \\
\text { fish meal }\end{array}$ & $1 \mathrm{R} / \mathrm{J}$ & $2 \mathrm{R} / \mathrm{J}$ & $\begin{array}{c}\text { Globale } \\
\text { sur l'ensemble } \\
\text { de la période } \\
\text { For the whole } \\
\text { period }\end{array}$ \\
\hline $\begin{array}{l}\text { 3. Expérimentale } \ldots \\
\text { Experimental }\end{array}$ & 10 & $\begin{array}{l}\text { Expérimental } \\
(93,5 \% \text { farine } \\
\text { de poisson }) \\
\text { fish meal }\end{array}$ & $2 \mathrm{R} / \mathrm{J}$ & $1 \mathrm{R} / \mathrm{J}$ & $\begin{array}{l}\text { Quotidienne } \\
\text { Daily }\end{array}$ \\
\hline
\end{tabular}

\section{TABLEAU 2}

CUD (moyenne et écart-type) de la matière sèche et de l'azote selon le plan expérimental (en p. 100).

Apparent digestibility (mean and standard deviation) of dry matter and nitrogen according to the experimental schedule.

\begin{tabular}{c|c|c|c}
\hline \hline \multirow{2}{*}{$\begin{array}{c}\text { Période } \\
\text { Period }\end{array}$} & $\begin{array}{c}\text { Fréquence des repas } \\
\text { (repas/jour) } \\
\text { Meal frequency } \\
\text { (meals/day) }\end{array}$ & $\begin{array}{c}\text { CUD } \\
\text { Apparent digestibility }\end{array}$ \\
\cline { 3 - 4 } & 1 & $\begin{array}{c}\text { Matière sèche } \\
\text { Dry matter }\end{array}$ & $\begin{array}{c}\text { Azote } \\
\text { Nitrogen }\end{array}$ \\
\cline { 3 - 4 } & 2 & $77,88 \pm 0,80$ & $89,03 \pm 0,59$ \\
& 1 & $78,55 \pm 1,20$ & $88,72 \pm 0,49$ \\
3 & 2 & $79,89 \pm 0,55$ & $90,26 \pm 0,33$ \\
& & $80,21 \pm 0,63$ & $90,46 \pm 0,46$ \\
\hline
\end{tabular}


Les calculs des coefficients d'utilisation digestive (CUD) sont effectués selon la relation formulée par MAYNARD \& LOOSLI (1969) et nous avons utilisé les méthodes statistiques classiques décrites par SNEdECOR \& COCHRAN (1967).

\section{Résultats}

Les valeurs moyennes des CUD obtenues selon le plan expérimental, sont rapportées dans le tableau 2. Aucune différence significative n'a pu être observée entre les valeurs de CUD de la matière sèche et de l'azote durant la seconde période.

La figure 1 rapporte l'évolution temporelle des CUD de la matière sèche et de l'azote durant la période expérimentale. On constate tout d'abord une évolution parallèle des valeurs des CUD de la matière sèche et de l'azote pour chaque fréquence de distribution journalière de l'aliment. Ce parallélisme illustre la bonne corrélation $(r>0,9)$ qui existe entre ces deux paramètres pour cet aliment.

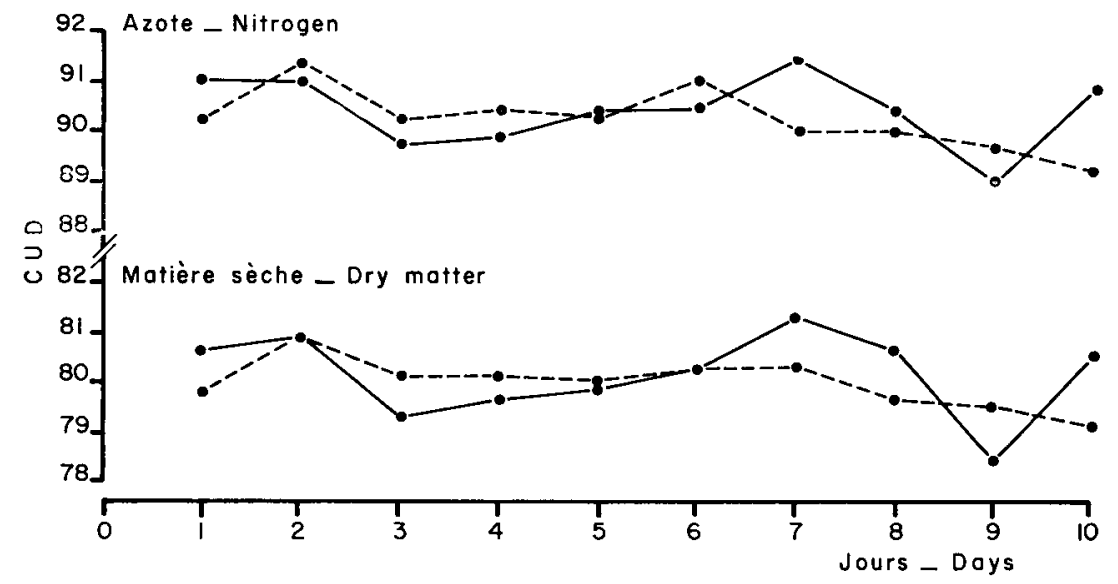

FIG. 1

Evolution temporelle des CUD de la matière sèche et de l'azote durant la période expérimentale.

Changes with time in the apparent digestibilities of dry matter and nitrogen during the experimental period.

2 repas/jour, -... - 1 repas/jour.

2 meals/day, - . - 1 meal/day.

De plus, au cours des premiers jours d'expérience, aucune variation journalière à tendance systématique n'est observée. La seule remarque que l'on puisse faire concerne les variations journalières plus fortes pour les lots recevant 2 repas/ jour que pour ceux ne recevant qu'un repas/jour. 


\section{Tableau 3}

Analyse des variances.

Analysis of variances.

\begin{tabular}{|c|c|c|c|c|}
\hline \multirow{2}{*}{$\begin{array}{l}\text { Source de } \\
\text { variation } \\
\text { Source of } \\
\text { variation }\end{array}$} & \multirow{2}{*}{ d.l. } & \multicolumn{2}{|c|}{$\begin{array}{l}\text { Carrés moyens } \\
\text { Mean squares }\end{array}$} & \multirow{2}{*}{$\begin{array}{c}\text { Coefficient } \\
\text { de corrélation } \\
\text { Coefficient } \\
\text { of correlation }\end{array}$} \\
\hline & & $\begin{array}{c}\text { Azote } \\
\text { Nitrogen }\end{array}$ & $\begin{array}{l}\text { Matière sèche } \\
\text { Dry matter }\end{array}$ & \\
\hline \multicolumn{5}{|l|}{$\begin{aligned} \text { A. } & 2 \mathrm{repas} / \text { jour } \\
2 & \text { Meals/day }\end{aligned}$} \\
\hline$\underset{\operatorname{Tank}}{\operatorname{Bac}} \ldots \ldots \ldots \ldots$ & 5 & $2,938^{*}$ & $4,076^{* *}$ & 0,962 \\
\hline $\begin{array}{l}\text { Jour } \quad \ldots \ldots \ldots \ldots \\
\text { Day }\end{array}$ & 9 & $3,128^{* * *}$ & $4,343 * *$ & 0,973 \\
\hline $\begin{array}{l}\text { Erreur } \\
\text { Error }\end{array}$ & 45 & 0,970 & 1,153 & 0,875 \\
\hline \multicolumn{5}{|l|}{$\begin{array}{l}\text { B. } 1 \mathrm{repas} / \text { jour } \\
1 \mathrm{Meal} / \text { day }\end{array}$} \\
\hline$\underset{\operatorname{Tank}}{\operatorname{Bac}} \ldots \ldots \ldots \ldots$ & 5 & $2,264 * *$ & $1,997 \mathrm{NS}$ & 0,965 \\
\hline $\begin{array}{l}\text { Jour } \\
\text { Day }\end{array}$ & 9 & $2,215^{k *}$ & $1,439 \mathrm{NS}$ & 0,900 \\
\hline$\underset{\text { Error }}{\text { Erreur }} \ldots \ldots \cdots \cdots$ & 45 & 0,548 & 0,967 & 0,852 \\
\hline \multicolumn{5}{|l|}{$\begin{array}{l}\text { C. Ensemble des données } \\
\text { All data }\end{array}$} \\
\hline $\begin{array}{l}\text { Classe }(\mathrm{A}, \mathrm{B}) \ldots \\
\text { Class }(A, B)\end{array}$ & 1 & $0,996 \mathrm{NS}$ & $0,891 \mathrm{NS}$ & - \\
\hline$\underset{\text { Tank }}{\text { Bac }} \ldots \ldots \cdots \cdots$ & 10 & $2,601^{k *}$ & $3,036 * *$ & 0,957 \\
\hline $\begin{array}{l}\text { Jour } \quad \cdots \cdots \cdots \cdots \\
\text { Day }\end{array}$ & 9 & $3,179 * *$ & $3,736 * *$ & 0,954 \\
\hline $\begin{array}{l}\text { Jour } \times \text { classe } \ldots \\
\text { Day } \times \text { class }\end{array}$ & 9 & $2,165^{* *}$ & $2,046 \mathrm{NS}$ & 0,904 \\
\hline $\begin{array}{l}\text { Erreur } \ldots \ldots \ldots \ldots \\
\text { Error }\end{array}$ & 90 & 0,759 & 1,060 & 0,862 \\
\hline
\end{tabular}

$\mathrm{NS}=$ non significatif - not significant.

$*=$ significatif à $\mathrm{p}=0,05$ - significant at $p=0.05$.

$*:=$ significatif à $\mathrm{p}=0,01$ - significant at $p=0.01$. 
Dans le tableau 3 sont rapportées les analyses de variances; celles-ci, décomposées en fonction des différentes sources de variation dont on peut estimer les effets (bacs, jours et erreur résiduelle) permettent de mettre en évidence :

- une absence totale d'effet de la fréquence de distribution journalière de l'aliment sur les valeurs des CUD de la matière sèche et de l'azote,

- une supériorité des carrés moyens des CUD relatifs aux lots recevant deux repas/jour par rapport aux lots ne recevant qu'un repas/jour. Ces différences de variances sont analogues pour le CUD de la matière sèche et celui de l'azote, leur coefficient de corrélation étant uniformément élevé.

\section{Discuision}

On sait que l'appétit des poissons est étroitement lié au volume de leur estomac (WARE, 1972) mais également à la température de l'eau (Grove, LoIzides \& NotT, 1978). Aussi avons-nous volontairement fixé le taux d'alimentation des poissons à 1 p. 100 de leur poids vif/jour. Ce choix reposait sur des considérations essentiellement d'ordre pratique. En effet, ce taux, bien inférieur au seuil de satiété permettait d'une part de nourrir les poissons rapidement (dans la limite de $30 \mathrm{mi}$ nutes pour l'ensemble des lots) surtout dans le cas où le repas était distribué en une fois et d'autre part de réduire les éventuels refus d'aliment.

S’il est bien établi que la collecte des fèces peut être effectuée dès le $3^{\circ}$ jour après un changement de composition du régime alimentaire (DE LA NouE et al., 1980), le délai de 5 jours observé ici tient compte de la température de l'eau et de son effet sur le temps de rétention des aliments dans le tube digestif des poissons (Possompes, Bergot \& Luduet, 1975). Les faibles valeurs de CUD de la matière sèche et de l'azote relevées dans la période d'accoutumance à l'aliment expérimental s'expliquent aisément par la persistance dans le tractus digestif de l'aliment antécédent (commercial) dont les CUD sont inférieurs.

Il est généralement admis qu'une fréquence de distribution supérieure à deux repas par jour n'apporte pas d'amélioration tant au niveau de la prise alimentaire qu'au niveau de la croissance ou de l'indice de consommation (Buterbaugh \& Willoughby, 1967 ; Grayton \& Beamish, 1977). Par contre, la distribution d'un repas par jour à des truites arc-en-ciel entraîne une consommation d'aliment plus faible et une vitesse de croissance des poissons légèrement inférieure (LuouET, Renou \& KaUshiK, 1981). Le présent travail montre que les valeurs des CUD de la matière sèche et de l'azote obtenues à partir de poissons recevant un ou deux repas par jour ne diffèrent pas significativement et que le changement de fréquence de distribution de l'aliment n'entraîne aucune évolution systématique des CUD dans les jours qui suivent. Le choix de l'une ou l'autre de ces fréquences de distribution d'aliment, dans le cas d'études de digestibilité chez le poisson, n'est donc qu'une question d'opportunité ; la variation d'erreur (bac, jour) plus faible dans le cas d'une fréquence de distribution d'un repas par jour pourrait même la rendre préférable. Ce point mériterait, toutefois, d'être développé.

Il convient cependant de noter que ces conclusions ont été tirées de l'étude de la digestibilité d'un aliment constitué exclusivement de farine de poisson dont on 
sait que la digestibilité est élevée. Des études analogues mais sur un aliment moins digestible apporteraient de précieux renseignements.

\section{Remerciements}

Les auteurs tiennent à remercier Mesdames Denise BlanC et Jeannine Breque ainsi que Monsieur B. Elhorga pour leur aide technique.

\section{Summary \\ Influence of a change in feeding frequency on diet digestibility in rainbow trout}

Dry matter and nitrogen (Nx6.25) digestibility values (table 2) obtained from rainbow trout fed once or twice a day an amount representing 1 p. 100 of their live weight/day, were not significantly different.

Changes in feeding frequency did not cause any systematic variation in the apparent digestibility (fig. 1).

The choice of one or the other method is a question of particular objective or convenience in fish digestibility studies. However, it should be pointed out that because of error variations (tank, day) which are lower in the case of one meal per day, this method is more advisable (table 3).

Key words : nutrition, frequency, meals, digestibility, trout.

Reçu en septembre 1983.

Accepté en janvier 1984.

\section{Références bibliographiques}

Andrews J.W., Murray M.W., DaVIS J.M., 1978. The influence of dietary fat levels and environmental temperature on digestible energy and absorbability of animal fat in catfish diets. J. Nutr., 108, 749-752.

Austreng E., 1978. Digestibility determination in fish using chromic oxide marking and analysis of contents from different segments of the gastro-intestinal tract. Aquaculture, 13, 265-272.

Bergot F., Breque J., 1983. Digestibility of starch by rainbow trout; effects of the physical state of starch and of the intake level. Aquaculture, 34, 203-212.

Bolin D.W., King R.P., Klosterman E.W., 1952. A simplified method for the determination of chromic oxide $\left(\mathrm{Cr}_{2} \mathrm{O}_{3}\right)$ when used as an index substance. Science, 116, 634-635.

Buterbaugh G., Willoughby H., 1967. A feeding guide for brook, brown and rainbow trout. Progve Fish-Cult., 29 (4), 6-11.

CASTLE E.J., 1956. The rate of passage of foodstuffs through the alimentary tract of goat. I. Studies on the adult animal fed on hay and concentrates. Br. J. Nutr., 10, 15-23.

Choubert G., De la Noue J., Luquet P., 1982. Digestibility in fish : Improved device for the automatic collection of feces. Aquaculture, 29, 185-189. 
Choubert G., De la Noue J., Luquet P., 1983. Un nouveau collecteur automatique quantitatif de fèces de poissons. Bull. Fr. Piscic., 288, 68-72.

Choubert G., Fauconneau B., Luquet P., 1982. Influence d'une élévation de la température de l'eau sur la digestibilité de la matière sèche, de l'azote et de l'énergie de l'aliment distribué à la truite arc-en-ciel (Salmo gairdneri, Rich.). Reprod. Nutr. Develop., 22, 941-949.

De la Noue J., Choubert G., Pagniez B., Blanc J.M., Luquet P., 1980. Digestibilité chez la truite arc-en-ciel (Salmo gairdneri) lors de l'adaptation à un nouveau régime alimentaire. Can. J. Fish. Aquat. Sci., 37, 2218-2224.

EDIN H., 1918. Orienterande försök över användberhetin av en pà led-kroppsprincipen grundad metod att bestämma en foderblandnings smältbarhed. Medd. Cent. Anst. Fors Väs. JordbrOmrâd (Stockholm), 165, 1-28.

Elliotr J.M., 1976. Energy losses in the waste products of brown trout (Salmo trutta L.). J. Anim. Ecol., 45, 561-580.

Grayton B.D., Beamish F.N.H., 1977. Effects of feeding frequency on food intake growth and body composition of rainbow trout (Salmo gairdneri). Aquaculture, 11, 159-172.

Grove D.J., Loizides L.G., NotT J., 1978. Satiation amount, frequency of feeding and gastric emptying rate in Salmo gairdneri. J. Fish. Biol., 12, 507-516.

Kaushik S.J., LuQuet P., 1976. Etude de la digestibilité des acides aminés de régimes à base de zéine chez la truite arc-en-ciel. Ann. Hydrobiol., 7, 11-19.

Luquet P., Renou P., Kaushik S.J., 1981. Influence du nombre de repas journaliers et du jeûne hebdomadaire sur la croissance chez la truite arc-en-ciel. Amn. Zootech., 30, 411-424.

Maynard L.A., Loosli J.K., 1969. Animal nutrition. 6th ed. Mc. Graw-Hill, New York, $613 \mathrm{p}$.

Pappas C.J., Tiemeier O.W., Deyoe C.W., 1973. Chromic sesqui-oxide as an indicator in digestion studies on channel catfish. Progve Fish-Cult., 35, 97-98.

Possompes B.P., 1973. Influence de la température sur les besoins en protéines, le transit alimentaire et la digestibilité chez la truite arc-en-ciel Salmo gaidneri Rich. Thèse $3^{e}$ cycle, Univ. Paris VI, 58 p.

Possompes B.P., Bergot P., Luquet P., 1975. Mise au point d'une méthode d'étude du transit gastro-intestinal chez la truite arc-en-ciel Salmo gairdneri Richardson : Influence du nombre de repas, des quantités ingérées et de la température d'acclimatation. Ann. Hydrobiol., 6, 131-143.

SNedecor G.W, Cochran W.G., 1967. Statistical methods. 6th ed. The Iowa state University press. Ames. 653 p.

Smith R.R., 1971. A method for measuring digestibility and metabolizable energy of fish feeds. Progve Fish-Cult., 33 , 132-134.

Smiti R.R., Peterson M.C., Allred A.C., 1980. Effect of leaching on apparent digestion coefficients of feedstuffs for Salmonids. Progve Fish-Cult., 42, 195-199.

WARE D.M., 1972. Predation by rainbow trout (Salmo gairdneri) : the influence of hunger, prey density and prey size. J. Fish. Res. Bd Can., 29, 1193-1201.

Windell J.T., Foltz J.W., SArokon J.A., 1978. Effect of fish size, temperature and amount fed on nutrient digestibility of a pelleted diet by rainbow trout Salmo gairdneri. Trans. Am. Fish. Soc., 107, 613-616.

ZIERLER K.L., 1958. A simplified explanation of the theory of indicator dilution for measurement of fluid flow and volume and other distributive phenomena. Bull. Johns Hopkins Hosp., 103, 199-217. 